\title{
Tiermodelle können die Krebsforschung revolutionieren
}

Wird menschliches Tumorgewebe in das Organ einer nackten Maus eingepflanzt, dem es entstammt, spricht man von einer orthotopen Implantation. Der Nutzen dieses Implantationsmodells zeigte sich bereits in einer Studie zur Therapie Cisplatin-resistenter Ovarialkarzinome. Durch die orthotope Implantation war es möglich, die histologischen, genetischen und epigenetischen Merkmale der menschlichen Tumoren sowie die Muster des Tumorwachstums zu reproduzieren. Nach Ansicht von Alberto Villanueva vom Institute of Biomedical Research of Bellvitge (IDIBELL) in Barcelona, Spanien, werden diese Tiermodelle eine Schlüsselrolle für die weitere Entwicklung der personalisierten Medizin bilden.

So konnte mit dieser Technik die Effektivität von Lurbinectedin (PM01183) das kürzlich von der Food and Drug Administration (FDA) als „Orphan Drug“ ge-

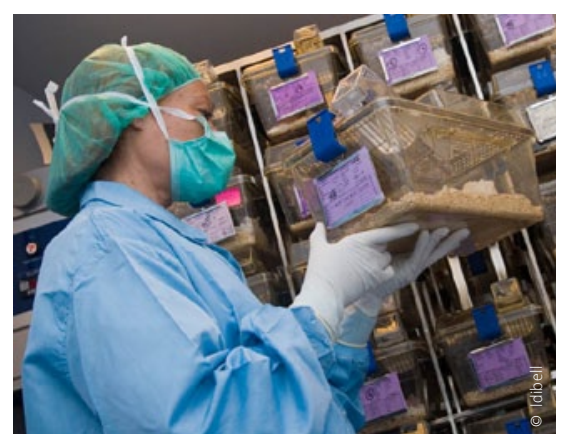

Tiermodelle sind in der onkologischen Forschung nach wie vor wichtig.

gen das Ovarialkarzinom zugelassen wurde, gezeigt werden. Das epitheliale Ovarialkarzinom stellt die fünfthäufigste Todesursache bei Frauen dar. Da die Diagnose oft erst zu einem fortgeschrittenen Zeitpunkt gestellt wird und häufig Resistenzen gegen Chemotherapeutika wie Cisplatin bestehen, ist die Überlebensrate gering.

Mithilfe der orthotopen Implantation kon von Tumorgewebe eines primären serösen Ovarialkarzinoms konnte nicht nur gezeigt werden, dass die Implantate ihr spezifischen humanen Eigenschaften bewahren. Mit ihrer Hilfe ließ sich auch das Ansprechen auf eine Monotherapie mit Lurbinectedin oder die Kombination von Lurbinectedin plus Cisplatin vorhersagen. Lurbinectedin inhibierte das Tumorwachstum, reduzierte die Proliferation des Tumors und induzierte schließlich die Apoptose der Tumorzellen.

Kim Jené

Vidal A et al. Lurbinectedin (PM01183), a New DNA Minor Groove Binder, Inhibits Growth of Orthotopic Primary Graft of Cisplatin-Resistant Epithelial Ovarian Cancer. Clin Cancer Res. 2012;18(19):5399-411.

\section{Wie sich Krebszellen vom Tumor befreien}

„Erhöht sich das Metastasierungspotenzial einer Krebszelle, geht die Adhäsion an normale Gewebestrukturen verloren. $\mathrm{Zu}$ dem entwickeln Krebszellen mit steigender Aggressivität die Fähigkeit, sich an spezifische Moleküle anzuheften und dort zu wachsen. Diese spezifischen Moleküle werden in gesundem Gewebe normal nicht gefunden, wohl aber an Orten, an denen sich Metastasen bilden“, erklärt Sangeeta Bhatia, Cambridge, MA/USA. Zellen verbleiben im Gewebeverband, weil sie über sogenannte Integrine an die extrazelluläre Matrix binden.

Im Mausmodell verglichen Bhatia und Kollegen die Adhäsionseigenschaften von vier Lungenkrebszelltypen: 1 . spät metastasierende primäre Lungentumoren, 2. nicht metastasierende primäre Lungentumoren, 3. Tumoren, die in regionäre Lymphknoten gestreut hatten, und 4. Tumoren, die Fernmetastasen gebildet hatten, z. B. in der Leber. Jeder dieser vier Zelltypen wurde etwa 800 verschiedenen Molekülpaaren der extrazellulärer Matrix ausgesetzt. Dann wurde die Bindung jedes Tumortyps an die Proteinpaare gemessen. Überraschenderweise verhielten sich die verschienden metastatische Zellen untereinander ähnlicher zueinander als zu ihren Primärtumoren. Besonders häufig binden metastatische Zellen an die extrazellulären Matrixmoleküle Fib- ronectin und Galectin-3. Interessant: Je aggressiver desto höher die Konzentration von Galectin-3. Hemmten die Forscher dann ein Integrins auf der Oberfläche der Krebszellen, dass mit Fibronectin und Galectin-3 interagierte führte dies bei den entsprechenden Mäusen zu einer verminderten Tumorstreuung.

Kim Jené

Reticker-Flynn NE et al. A combinatorial extracellular matrix platform identifies cell-extracellular matrix interactions that correlate with metastasis. Nat Commun. 2012 Oct 9 [epub ahead of print]

\section{kurz notiert}

\section{DETECT-III-Studie angelaufen}

DETECT-III-Studie ist eine multizentrische, randomisierte Phase-III-Studie zum Vergleich einer Standardtherapie allein versus eine Standardtherapie plus Lapatinib bei Patientinnen mit initial HER2-negativem metastasiertem Brustkrebs und HER2-positiven zirkulierenden Tumorzellen.

Rationale ist die Evaluation der Wirksamkeit von Lapatinib bei Patientinnen mit metastasiertem Brustkrebs, welche HER2-positive zirkulierende Tumorzellen (CTC) aufweisen, obwohl der Primärtumor und/oder Gewebepro- ben einer metastatischen Läsion auf ihren HER2-Status getestet wurden und HER2-Negativität zeigten. Primärer Endpunkt ist das progressionsfreie Überleben.

Studienleitung:

Sponsor der Studie ist die Heinrich-Heine Universität Düsseldorf, vertreten durch Prof. Dr. Wolfgang Janni, Klinische Studienleiterin ist Prof. Dr. Tanja Fehm, Tübingen.

Weitere Informationen: http://www.detect-studien.de 